\title{
Effect of a near-cathode sheath on heat transfer in high-pressure arc plasmas
}

\author{
He-Ping $\mathrm{Li}^{1,3}$ and M S Benilov ${ }^{2}$ \\ ${ }^{1}$ Department of Engineering Physics, Tsinghua University, Beijing 100084, People's \\ Republic of China \\ ${ }^{2}$ Departamento de Fisica, Universidade da Madeira, Largo do Minicípio, 9000 Funchal, \\ Portugal \\ E-mail: liheping@tsinghua.edu.cn
}

Received 27 November 2006, in final form 13 January 2007

Published 16 March 2007

Online at stacks.iop.org/JPhysD/40/2010

\begin{abstract}
Numerical simulation of a high-pressure arc discharge has been performed with a self-consistent modelling of most of the components, including the electrodes, and the interactions between them. In particular, the arc column and the cathodic part of the discharge are simulated by means of a two-temperature hydrodynamic model and of a model of nonlinear surface heating, respectively. Simulation results are given for a free-burning arc in atmospheric-pressure argon in the range of arc currents from 10 to $200 \mathrm{~A}$. It is found that the electric power deposited into the near-cathode layer is transported not only to the cathode but also to the arc column, an effect that cannot be described by the local thermodynamic equilibrium (LTE) model. The electron enthalpy transport substantially exceeds the net contribution of thermal conduction by the electrons and heavy particles and is thus the dominating mechanism of energy transfer from the near-cathode layer to the arc column. The predicted gas temperatures along the arc axis in the arc column using the LTE model are much higher than the calculated electron and heavy-particle temperatures $(\sim 1000-2000 \mathrm{~K}$ or higher $)$ under the same operation conditions using the non-equilibrium model with the consideration of the near-cathode sheath for the cases studied in the present paper. Studies on the influences of the cathode shapes and metal vapour contaminations on the arc characteristics will be conducted in future work.
\end{abstract}

(Some figures in this article are in colour only in the electronic version)

\section{Introduction}

Gas discharge plasmas operated at different pressure levels have been widely used in different fields, such as plasma spraying, propulsion, cutting, welding, lighting, plasma-aided chemical vapour deposition, waste treatment and so on [1]. Many studies have been conducted for investigating the effects of different conditions, such as the operation parameters, the plasma-working gases, the electrode materials and shapes and the applied magnetic fields on the arc characteristics and complex interactions between plasmas and electrodes (or cold walls) (e.g. [1-6] and references therein). The high-pressure electric arc consists of a set of electrodes (cathode and anode),

3 Author to whom any correspondence should be addressed. arc column and near-electrode regions [7]. The arc column region represents the main body of the arc, while the nearelectrode region refers conventionally to a non-equilibrium plasma region separating the electrode surface from the arc column. Although the near-electrode region is a very thin layer characterized by steep gradients of temperature, particle density and potential, many complicated physical processes are involved in this region [8].

An adequate account of near-electrode space-charge sheaths has always been a subject of primary concern in numerical modelling of low-pressure gas discharges. The situation is very different as far as high-pressure arc discharges are concerned: numerical models published up to now either take into account near-electrode sheaths by means of very crude - and in many cases, quite arbitrary-assumptions, or 
neglect their presence altogether. This difference may be attributed, at least partially, to the fact that the near-electrode sheath in high-pressure arc discharges is not accessible to direct experimental observation, and even indirect experimental data are quite difficult to be obtained.

However, detailed experimental investigations performed recently (e.g. [9-16] and references therein) have unambiguously confirmed the existence of space-charge sheaths adjacent to cathodes in high-pressure arc plasmas, with sheath voltages that may be as high as $50 \mathrm{~V}$. The experiment has also unambiguously confirmed the theory of plasma-cathode interaction (e.g. [14,17-29] and references therein) in which the concept of near-cathode space-charge sheath is of central importance. In such a situation, it is appropriate to try to understand how an adequate account of the near-cathode space-charge sheath would affect the results of numerical modelling of the arc discharge on the whole.

The best way to do so would be, of course, to perform a modelling of the whole discharge region, from the cathode to the anode, without assuming the plasma to be quasi-neutral, as is common in modelling of low-pressure discharges. However, for high-pressure arc discharges this task is computationally very intense, given a high degree of quasi-neutrality of the bulk plasma and very small volume occupied by the sheaths. In the present work, this task is performed by means of a separate treatment of the near-cathode sheath and the arc column with an appropriate matching. A free-burning argon arc in a wide range of arc current, from 10 to $200 \mathrm{~A}$, is treated. It is shown that the electric power deposited into the near-cathode layer is transported not only to the cathode surface, heating it and thus ensuring the necessary level of thermionic emission, but also to the arc column, thus providing additional heating of the bulk of the arc. By comparing with the results given by a local thermodynamic equilibrium (LTE) model, it is confirmed that although the LTE model can be employed to predict general features of the arc column, it does not correctly describe the process of energy exchange between the near-cathode layer and the arc column.

\section{The model}

\subsection{Calculation domain}

In previous studies, a high-intensity, free-burning arc was usually employed to investigate the arc characteristics (e.g. $[7,30]$ and references therein). In this paper, this configuration is also used to study the heat transfer features of the arc, where for simplifications in the near-cathode sheath model [8], the cathode tip is truncated, which was also employed in actual applications (e.g. in the gas-metal arc welding [6]). The calculation domain includes the electrodes (cathode and anode), near-cathode region and the arc column region, as shown in figure 1 with geometrical dimensions and the origin of the axis located at point $J$. The near-anode region is not considered in this study, but a harmonic mean value of the electric conductivity is employed to ensure the current passage from the arc column to the anode body [30]. The length and diameter of the tungsten rod cathode are $10.0 \mathrm{~mm}$ and $2.0 \mathrm{~mm}$, respectively. The distance between the cathode frontal surface and the anode surface is $10.0 \mathrm{~mm}$. The outer edge of the

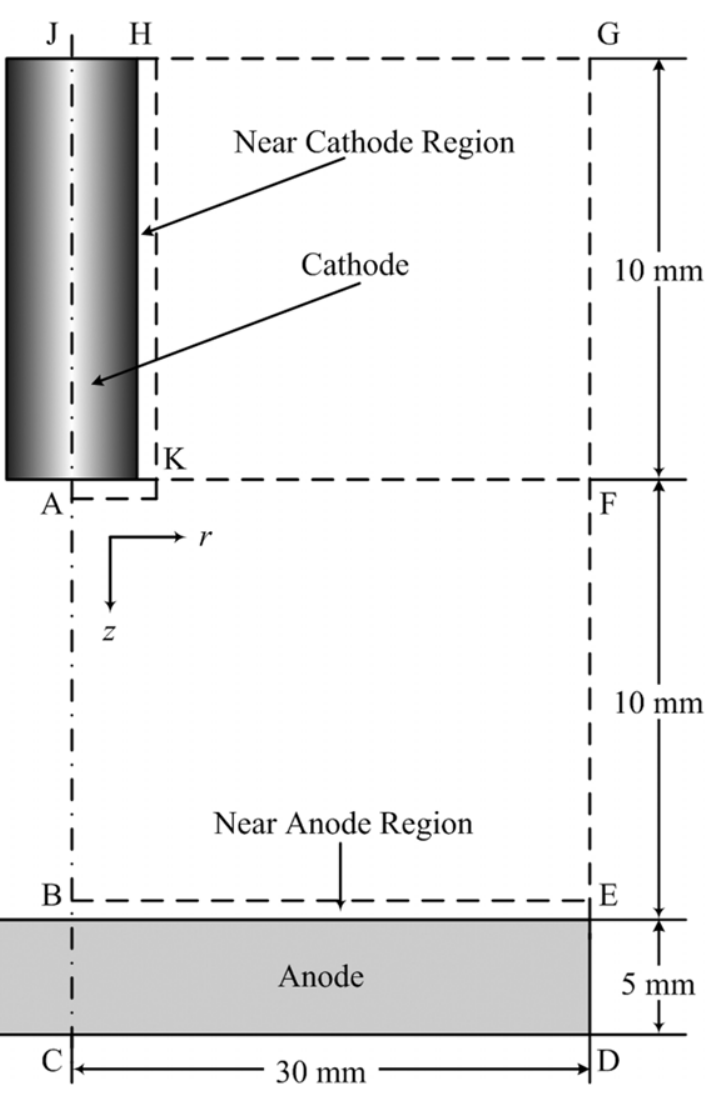

Figure 1. Calculation domain.

calculation domain is located $30.0 \mathrm{~mm}$ away from the arc axis in the radial direction, so that the fluid flow near the outer edges can be neglected. The problem is stationary and axially symmetric.

In this work, each of the sub-domains (the arc column, the near-cathode plasma layer, the cathode and the anode) is described by means of a separate model, with a proper coupling between different sub-domains. An important aspect of this coupling is as follows. The energy of electrons in the near-cathode layer of a high-pressure arc discharge is very high, which is a consequence of very high values of the electric field. Therefore, the energy of electrons leaving the near-cathode layer and entering the arc column is much higher than the temperature of heavy particles. Hence, an adequate model of arc column cannot be a LTE one: it must take into account a difference between the electron and heavyparticle temperatures. This applies even to high-current arcs, in which the difference between the electron and heavy-particle temperatures in the bulk of the arc is negligible [31]: there is a part of the arc column adjacent to the near-cathode layer in which the temperatures are essentially different, and a correct description of this part is critical for understanding the heat exchange between the near-cathode layer and the arc column.

In what follows, we describe the models used for the description of each of the sub-domains.

\subsection{Arc column}

In the present paper, we assume that: (1) in the arc column, the argon plasma system deviates from the LTE state but is 
in the local chemical equilibrium (LCE) state, and the twotemperature (2-T) model can be employed; (2) the plasma flow is laminar, gravity is neglected as a minor effect, the viscous dissipation and the work of the pressure gradient are small effects due to low values of the Mach number and are neglected as well; (3) the plasma is optically thin and (4) the contamination of metal vapour originating from the electrodes is not considered in this study.

Based on the foregoing assumptions, the governing equations in the cylindrical coordinates $(r, z)$ are listed as follows.

\section{Continuity equation:}

$$
\frac{1}{r} \frac{\partial}{\partial r}\left(r \rho v_{r}\right)+\frac{\partial}{\partial z}\left(\rho v_{z}\right)=0 .
$$

Momentum conservation equations:

$z$-direction (axial):

$$
\begin{gathered}
\rho\left(v_{r} \frac{\partial v_{z}}{\partial r}+v_{z} \frac{\partial v_{z}}{\partial z}\right)=-\frac{\partial p}{\partial z}+\frac{1}{r} \frac{\partial}{\partial r}\left[\mu r\left(\frac{\partial v_{z}}{\partial r}+\frac{\partial v_{r}}{\partial z}\right)\right] \\
+\frac{\partial}{\partial z}\left(2 \mu \frac{\partial v_{z}}{\partial z}\right)+j_{r} B_{\theta} .
\end{gathered}
$$

$r$-direction (radial):

$$
\begin{aligned}
& \rho\left(v_{r} \frac{\partial v_{r}}{\partial r}+v_{z} \frac{\partial v_{r}}{\partial z}\right)=-\frac{\partial p}{\partial r}+\frac{\partial}{\partial r}\left(2 \mu \frac{\partial v_{r}}{\partial r}\right) \\
& +\frac{\partial}{\partial z}\left[\mu\left(\frac{\partial v_{r}}{\partial z}+\frac{\partial v_{z}}{\partial r}\right)\right]+\frac{2 \mu}{r}\left(\frac{\partial v_{r}}{\partial r}-\frac{v_{r}}{r}\right)-j_{z} B_{\theta} .
\end{aligned}
$$

Energy conservation equation for electrons:

$$
\begin{aligned}
\nabla \cdot & \left(\frac{5}{2} n_{\mathrm{e}} k_{\mathrm{B}} T_{\mathrm{e}} \vec{v}\right)=\nabla \cdot\left(k_{\mathrm{e}} \nabla T_{\mathrm{e}}\right)+\vec{j} \cdot \vec{E}-U_{\mathrm{r}} \\
& +\frac{5}{2} \frac{k_{\mathrm{B}}}{e}\left(\vec{j} \cdot \nabla T_{\mathrm{e}}\right)-\dot{E}_{\mathrm{el}} .
\end{aligned}
$$

Energy conservation equation for heavy particles:

$$
\begin{aligned}
\nabla \cdot\left\{\left[\frac{5}{2}\left(n_{\mathrm{a}}+n_{\mathrm{i}}\right) k_{\mathrm{B}} T_{\mathrm{h}}+n_{\mathrm{i}} E_{\mathrm{i}}\right] \vec{v}\right\} \\
=\nabla \cdot\left[\left(k_{\mathrm{a}}+k_{\mathrm{i}}+k_{\mathrm{r}}\right) \nabla T_{\mathrm{h}}\right]+\dot{E}_{\mathrm{el}} .
\end{aligned}
$$

where the subscripts $\mathrm{h}$, e, a and i represent heavy particle, electron, atom and singly ionized ion, respectively. $\rho, \mu$ and $p$ are the mass density, dynamic viscosity and pressure of the gas, $v_{r}$ and $v_{z}$ are the components of the velocity vector $\vec{v}$ in $r$ - and $z$-directions, respectively. $e$ and $k_{\mathrm{B}}$ are the elementary charge and Boltzmann constant. $E_{\mathrm{i}}$ is the ionization energy. $n, k, T$ and $U_{\mathrm{r}}$ are particle number density, thermal conductivity, temperature and radiation power per unit volume. All the thermodynamic and transport properties of argon plasmas are functions of the temperatures $\left(T_{\mathrm{e}}\right.$ and $\left.T_{\mathrm{h}}\right)$ at atmospheric pressure. $\vec{j}$ and $\vec{E}$ are the current density vector and the electric field vector, respectively. $B_{\theta}$ is the self-induced magnetic field in the circumferential direction (or $\theta$-direction) calculated as $B_{\theta}\left(\mu_{0} / r\right) \int_{0}^{r} j_{z} \xi \mathrm{d} \xi$, where $\mu_{0}$ is the permeability of vacuum. $\dot{E}_{\mathrm{el}}$ is the energy transferred in elastic collisions between electrons and heavy particles, which is expressed as

$$
\dot{E}_{\mathrm{el}}=\left(2 m_{\mathrm{e}} / m_{\mathrm{h}}\right)\left(\bar{v}_{\mathrm{ea}}+\bar{v}_{\mathrm{ei}}\right) n_{\mathrm{e}}\left[(3 / 2) k_{\mathrm{B}}\left(T_{\mathrm{e}}-T_{\mathrm{h}}\right)\right],
$$

where $\bar{v}_{\mathrm{ei}}$ and $\bar{v}_{\mathrm{ea}}$ are the average collision frequency between electrons and ions, and between electrons and atoms, respectively [32,33], $m_{\mathrm{e}}$ and $m_{\mathrm{h}}$ are the mass of an electron and heavy particle, respectively.

\section{Electric potential equation:}

$$
\nabla \cdot(\sigma \nabla \phi)=0
$$

where $\sigma$ is the electric conductivity of plasmas, $\phi$ is the electric potential related to the electric field as $\vec{E}=-\nabla \phi$ and the current density is expressed as $\vec{j}=\sigma \vec{E}$.

\subsection{The cathode body and near-cathode plasma layer}

The cathode body and near-cathode plasma layer are simulated by means of the model of nonlinear surface heating. This model represents a basis of the modern theory of plasmacathode interaction in high-pressure arc discharges (e.g. [14, 17-29] and references therein) and has been confirmed by detailed experimental investigation (e.g. [9-16] and references therein).

The model of nonlinear surface heating is based on the assumption that the energy flux to the cathode surface is generated in the near-cathode plasma layer, a contribution of the energy flux from the arc column being absent or negligible, and that the near-cathode voltage drop considerably exceeds the voltage in the expansion region. In the framework of these assumptions, the cathodic part of the high-pressure arc discharge is to a first approximation not affected by the arc column and may be simulated independently. An investigation of the validity of this approach represents one of the objectives of this work.

In this work, the model of nonlinear surface heating is used along the lines of [34] with modifications summarized in [35] (Some additional theoretical material on this model can be found on the Internet [36].) The cathode is assumed to be made of tungsten.

\subsection{The anode body}

Inside the anode body, only the heat conduction equation and current continuity equation are used, i.e.

$$
\begin{gathered}
\nabla \cdot\left(k_{\mathrm{m}} \nabla T\right)+\vec{j} \cdot \vec{E}=0, \\
\nabla \cdot\left(\sigma_{\mathrm{m}} \nabla \phi\right)=0,
\end{gathered}
$$

where $k_{\mathrm{m}}$ and $\sigma_{\mathrm{m}}$ are the thermal conductivity and electric conductivity of the anode material (copper), physical properties of which are taken from [37].

The additional energy flux from the arc column to the anode surface is

$$
q_{\mathrm{pa}}=|\vec{j}|\left(W_{\mathrm{a}}+2.5 \frac{k_{\mathrm{B}}}{e} T_{\mathrm{e}}\right)
$$


with a similar idea as that presented in [30], where $W_{\mathrm{a}}$ is the work function of the anode material $\left(W_{\mathrm{a}}=4.65 \mathrm{~V}\right.$ is employed in this study [30]). After multiplying by the surface area of the pertinent near-surface control volume, and then, dividing by its volume, $q_{\mathrm{pa}}$ will be added to the near-surface control volume on the anode side as the additional source term in the energy equation (8).

\subsection{Boundary conditions}

\subsubsection{Conditions on external boundaries of the calculation} domain. The conditions along the external boundaries of the calculation domain are as follows: at the axis of symmetry of the system, the axially symmetric conditions are employed for the independent variables; at the bases of the cathode $(\mathrm{JH})$ and anode (CD), a uniform temperature $(293 \mathrm{~K})$ is assumed, and a uniform electric potential $(\phi=0)$ is specified at the base of the anode (CD); at the outer edges of the calculation domain (DEFGH), zero velocity, uniform temperature (293 K) and zero normal derivative of the potential are specified. The potential of the cathode is constant and is calculated as a part of this solution.

\subsubsection{Conditions of matching of solutions in different sub-} domains. Inner boundary conditions must be established at the interfaces between the cathode and the near-cathode layer, between the arc column and the near-cathode layer and between the arc column and the anode. The boundary conditions on the cathode surface represent a part of the model of nonlinear surface heating: the density of energy flux coming to the surface from the plasmas equal to the density of the heat flux removed by thermal conduction from the surface into the bulk of the cathode. The boundary conditions on the interface between the near-cathode layer and the arc column have been established in [8]: the electron and heavy-particle temperatures and the normal component of the electric current density are continuous. (As far as the heavy-particle temperature in the arc column is concerned, this means that it equals the local temperature of the cathode surface.)

At the arc-anode interface, the conditions of continuous fluxes for energy transfer and current transfer are employed for heavy-particle temperature and electric potential. Zero gradient of electron temperature is imposed for determining the electron temperature. Volumes of sheaths are neglected while calculating the arc column.

\section{Modelling results and discussions}

\subsection{Numerical solution}

The cathode body and the near-cathode plasma layer are calculated by means of the free online tool for simulation of the diffuse mode of current transfer developed on the basis of the model of nonlinear surface heating [36]. Readers desiring the detailed descriptions on the assumptions and physical/mathematical models corresponding to this free online tool can refer to [8]. The near-cathode voltage drop $U$ is adjusted in such a way that the arc current takes a desired value. Then, the output data produced by the tool (distributions along the cathode surface of the local electron temperature in

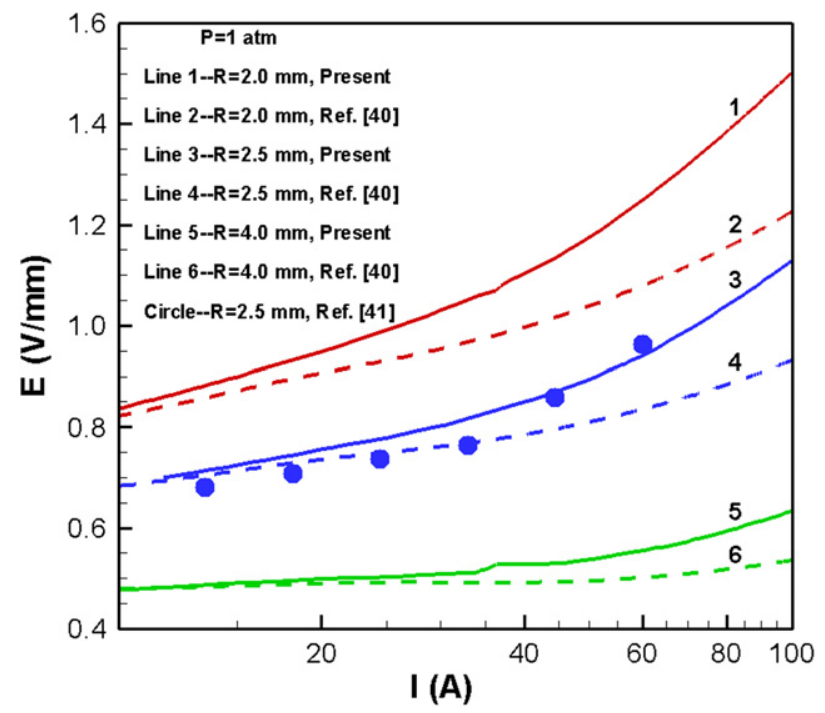

Figure 2. Electric field in a cylindrical column of a wall-stabilized $\mathrm{dc}$ arc in argon at atmospheric pressure [41]: experiment, present work and [40]: modelling.

the near-cathode layer $\left(T_{\mathrm{e}}\right)$, of the local temperature of the cathode surface $\left(T_{\mathrm{w}}\right)$ and of the local density of the net current from the plasma to the cathode surface $(j))$ are employed as the inner boundary conditions for the modelling of the arc column region. After this, the problems describing the arc column and the anode body are solved.

The governing equations in the arc column are solved using a newly developed computer code, which represents a new version of the Flow Analysis Simulation Tool of TwoDimensional (FAST-2D) [38]. The original FAST-2D code is a general-purpose computer program for simulating twodimensional (2D) incompressible flows with constant fluid thermodynamic and transport properties and can be employed to treat the problem with complex boundary geometry based on the SIMPLE-like algorithm [38,39]. The new version used in this study allows one to simulate the heat transfer and fluid flow under thermal plasma conditions with variable thermodynamic and transport properties and with or without electromagnetic fields. An important advantage of this code is that it is not commercial software and thus one has access to and can control every computational detail. In this study, a $76(z) \times 46(r)$ non-uniform mesh is employed for simulating the arc characteristics in the arc column region and the anode body, with the distance between the first grid point on the plasma side and the electrode surface being $\sim 0.1 \mathrm{~mm} \mathrm{[30].}$

In order to validate the revised computer code and the mathematical model for 2-T modelling of thermal plasmas, a one-dimensional problem of a cylindrical column of a wallstabilized dc arc discharge in atmospheric-pressure argon was solved; a kind of benchmark problem. While dealing with this problem, the variations of the parameters in the axial direction are neglected and the energy conservation equations of electrons and heavy particles given in section 2.2 are simplified similarly to how it was done in [40]. In figure 2, the values of the calculated axial electric field are shown along with the experimental data taken from [41]. Also shown are the results of the modelling [40], which has been performed for a 2-T plasma without the assumption of ionization equilibrium 
but neglecting the radiation losses. Deviations between the data calculated in the present work and the experimental data are smaller than $6 \%$. At currents not too high, the present results are in agreement also with the modelling results [40]. One can conclude that the mathematical model and the computer code used in this study are valid.

\subsection{Non-equilibrium features of the arc plasmas}

Non-equilibrium effects in the arc column come into play at low currents (e.g. [40] and references therein). Besides, nonequilibrium effects are always pronounced near the electrodes and (cold) walls and in the fringes of free-burning arcs [31]. For revealing this non-equilibrium feature in the arc column region, the 2-T model, which is described in section 2, and the LTE model, which is employed in previous papers (e.g. [30, 42]), are used to predict the temperature distributions in the arc column region. In figure 3 , the electron and heavy-particle temperature profiles along the axis of symmetry in the arc column region are illustrated. Figure 3 shows that (1) not only in the regions near the electrodes, but also in the arc core region, the plasma deviates significantly from the LTE state for the cases with low arc currents; (2) with increasing arc current, the variations of the electron temperature in the downstream region of the cathode along the axis of symmetry become small; (3) for the cases with larger arc currents, the discrepancies between the electron and heavy-particle temperatures become less significant due to the more efficient energy exchange processes between electrons and heavy particles, which leads to a LTE plasma state in the core region of the arcs for higher arc currents and (4) even at high arc currents, where deviations between the electron and heavy-particle temperatures in the arc column are insignificant and the arc column plasma is in LTE, the temperatures predicted by the non-LTE model are significantly lower than the temperature predicted by the LTE model.

The latter effect is illustrated in figure 4 , in which are shown radial distributions of the electrical conductivity of the plasma, of the current density, of the heavy-particle and electron temperatures and of the temperature calculated in the LTE approximation, in the mid-plane between the frontal surface of the cathode and the anode $(z=15.0 \mathrm{~mm})$ with arc current $I=100 \mathrm{~A}$. The $2-\mathrm{T}$ model predicts higher electron temperatures and, consequently, higher electrical conductivities in the arc fringes. Therefore, the current density increases in the arc fringes and correspondingly decreases near the arc core region, leading to a lowering of the temperatures in the arc core region. Note that this effect was also reported in other works dedicated to non-equilibrium modelling of highpressure arc discharges; see, e.g. [43-45].

\subsection{Energy fluxes from the near-cathode plasma region}

According to the nonlinear surface heating model presented in [24], the electric power deposited into the near-cathode layer is transferred to heat the cathode body, and is also transferred to heat the gas in the arc column region (cf equation (13) in [24]). The density of the net energy flux to the cathode surface generated in the near-cathode region can be expressed as [24]

$$
q_{\mathrm{nc}}=q_{\mathrm{i}}+q_{\mathrm{e}}-q_{\mathrm{em}}-q_{\mathrm{r}},
$$
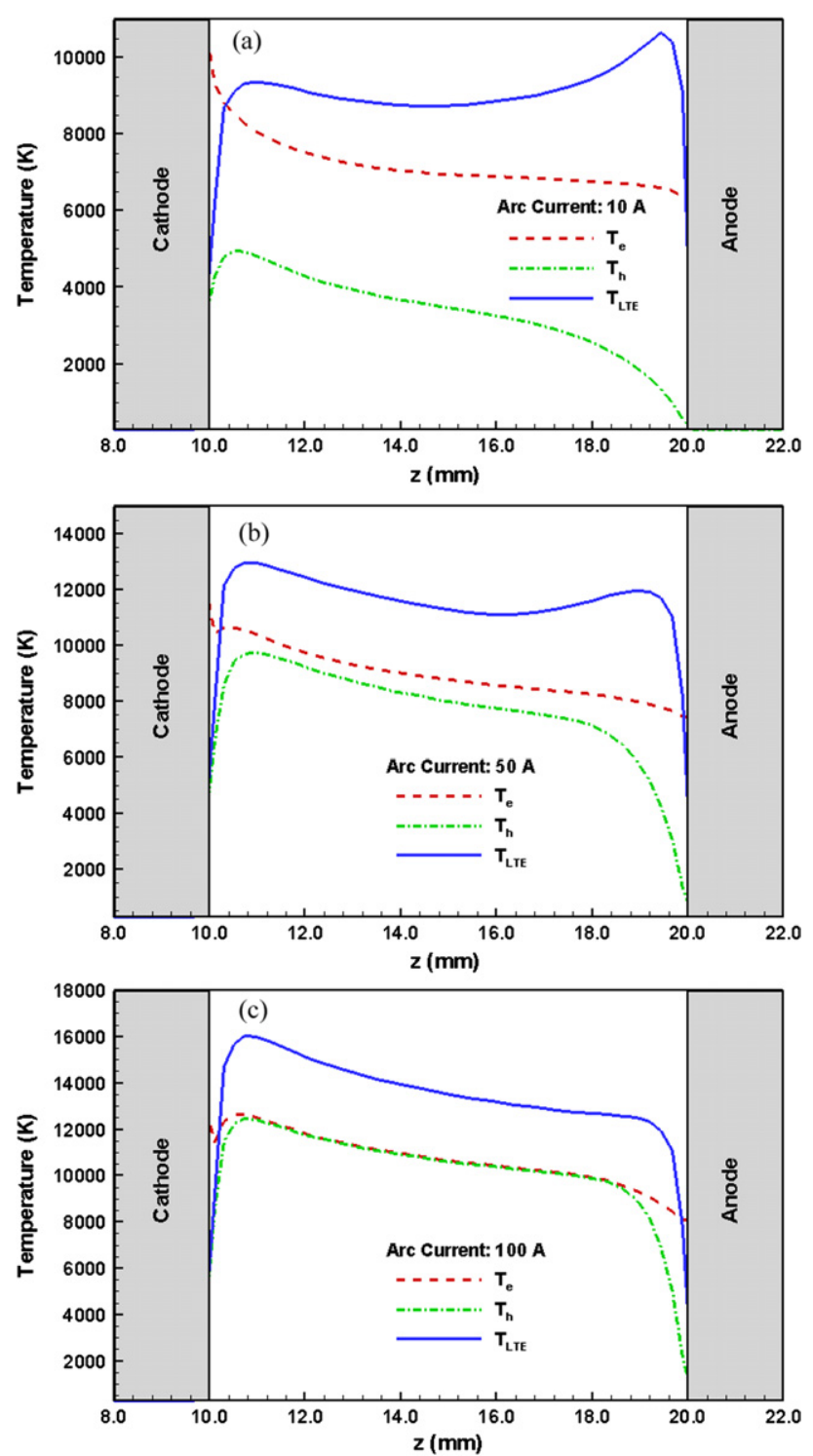

Figure 3. Profiles of the gas temperatures along the arc axis predicted using the LTE model $\left(T_{\mathrm{LTE}}\right)$, and the electron $\left(T_{\mathrm{e}}\right)$ and heavy-particle $\left(T_{\mathrm{h}}\right)$ temperatures using the 2 -T model for different arc currents.

where $q_{\mathrm{i}}$ and $q_{\mathrm{e}}$ represent the densities of energy fluxes delivered to the cathode surface by ions and by fast electrons, respectively, while $q_{\mathrm{em}}$ and $q_{\mathrm{r}}$ are the densities of losses of energy by the cathode surface due to thermionic emission and radiation, respectively. In the framework of the 2-T model, the density of the energy flux transferred to the near-cathode region from the arc column can be expressed as

$$
q_{\mathrm{np}}=q_{\mathrm{hc}}+q_{\mathrm{ec}}+q_{\mathrm{enthalpy}},
$$

where $q_{\mathrm{hc}}, q_{\mathrm{ec}}$ and $q_{\text {enthalpy }}$ represent the densities of energy fluxes due to heat conduction of heavy particles, electrons and of the electron enthalpy transport, which are expressed as $q_{\mathrm{hc}}=k_{\mathrm{h}} \partial T_{\mathrm{h}} / \partial n, \quad q_{\mathrm{ec}}=k_{\mathrm{e}} \partial T_{\mathrm{e}} / \partial n$,

$q_{\text {enthalpy }}=-\frac{5}{2} \frac{k_{\mathrm{B}}}{e} j_{\mathrm{n}} T_{\mathrm{e}}$,

where $n=z$ on the front surface of the cathode (AK in figure 1) and $n=r$ on the lateral surface (KH in figure 1). For the energy 

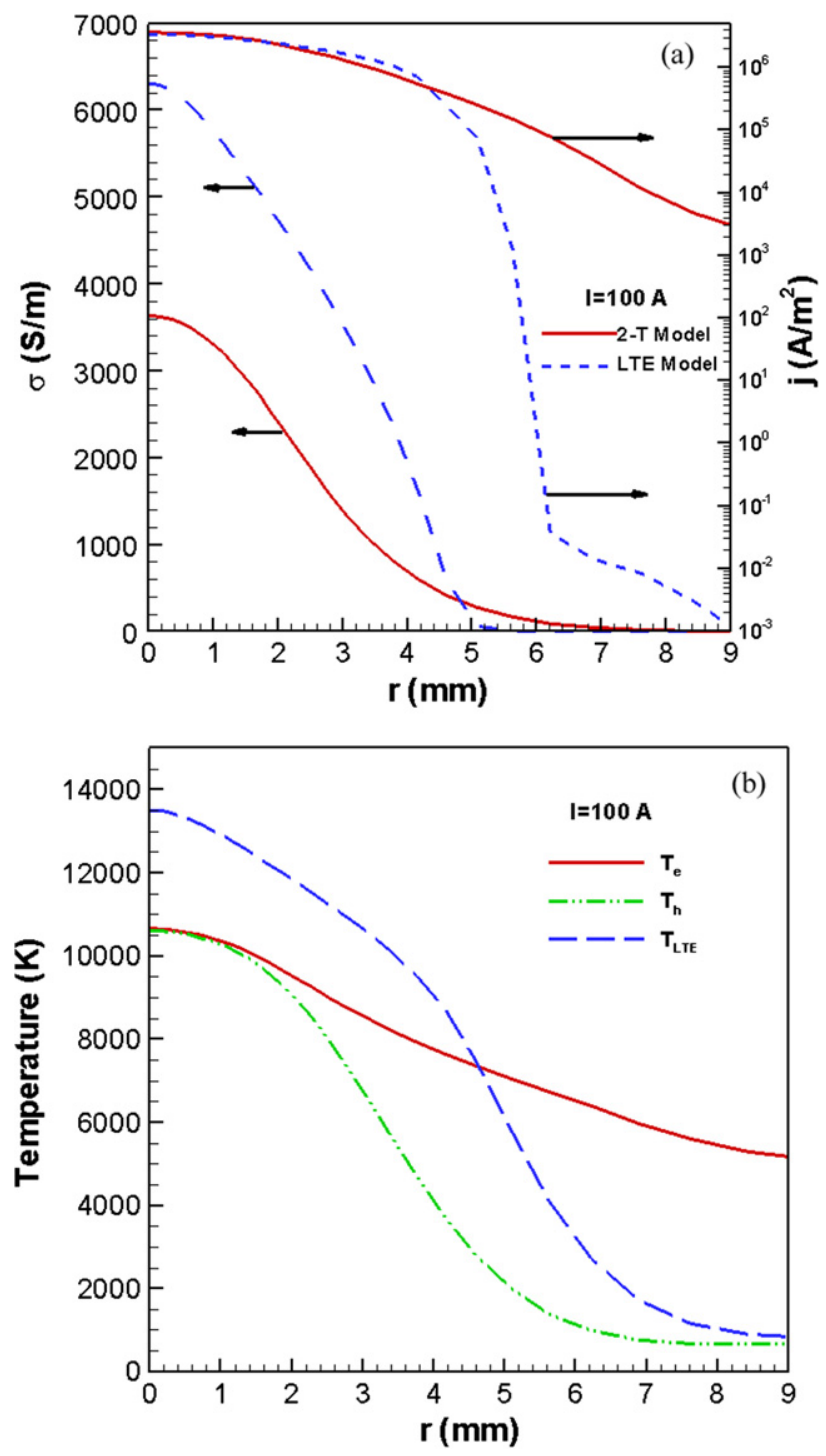

Figure 4. Distributions of the electrical conductivity, current density and temperatures along the radial direction in the mid-plane $(z=15.0 \mathrm{~mm})$ between the cathode frontal surface and the anode surface for arc current $I=100$ A.

exchange between the cathode body and the near-cathode layer, described by equation (11), the positive energy flux densities represent the energy entering the cathode body, while for the energy exchange between the near-cathode layer and the arc column, described by equation (13), the positive energy flux densities represent the energy delivered to the near-cathode layer from the arc column.

The distributions of the densities of energy fluxes along the cathode surface delivered to the cathode, $q_{\mathrm{nc}}$, and of the energy fluxes transferred from the arc column to the nearcathode layer, $q_{\mathrm{np}}$, and its components $q_{\mathrm{hc}}, q_{\mathrm{ec}}$ and $q_{\text {enthalpy }}$, are illustrated in figure 5 for the case with arc current $I=30 \mathrm{~A}$. For comparison, the predicted energy flux density delivered to the near-cathode layer using a LTE model $\left(q_{\mathrm{np}, \mathrm{LTE}}\right)$ is also shown in this figure. Since the radius of the cathode rod is $1.0 \mathrm{~mm}$, the range $0 \leqslant r+z \leqslant 1 \mathrm{~mm}$ in figure 5 corresponds to the front surface, while the range $r+z \geqslant 1 \mathrm{~mm}$ corresponds to the lateral surface. Figure 5 shows that (1)

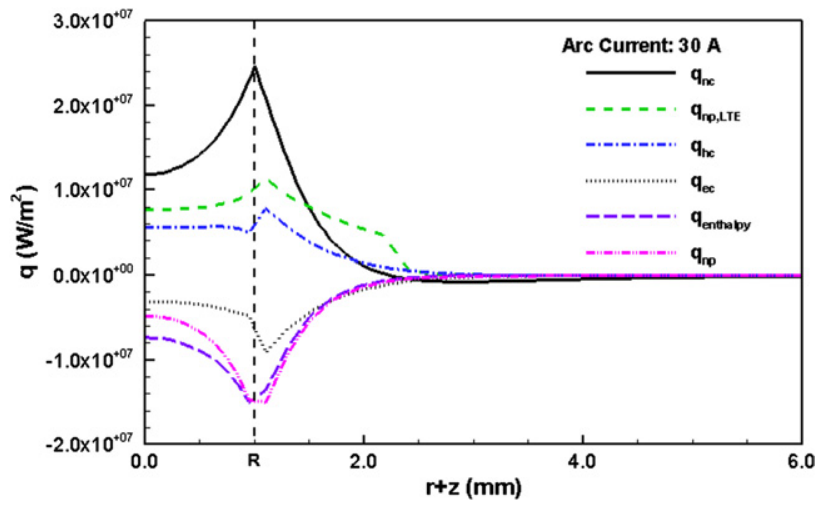

Figure 5. Distributions of the energy flux densities along the cathode surface for arc current $I=30$ A.

the distribution along the cathode surface of the density of energy flux from the plasma to the cathode has a maximum at the edge of the front surface. Note that the temperature of the cathode surface, the density of electric current from the plasma to the cathode surface, the electron temperature in the near-cathode layer, etc, also attain maxima at the edge of the front surface. The minimum or maximum points of $q_{\mathrm{np}}$ and its components do not exactly coincide with the edge of the cathode surface, but this displacement is a numerical effect (there was no knot of the numerical grid at the edge); (2) in the framework of the 2-T model, the heavy particles always deliver energy from the arc column to the near-cathode layer (by thermal conduction), while the electrons deliver energy from the near-cathode layer to the arc column (both by thermal conduction and enthalpy transport); (3) the electron energy transport dominates, resulting in a negative value of $q_{\mathrm{np}}$, which means that a part of the energy deposited in the near-cathode region is transported to the arc column and not the other way round; a very important result that confirms the assumption of the cathodic part of the discharge being independent of the arc column (this assumption lies at the basis of the model of nonlinear surface heating as discussed in section 2.3 above) and (4) in contrast to the energy flux densities calculated using the 2-T model, the density of the energy flux predicted using the LTE model, $q_{\text {np,LTE}}$, is always positive, which means that the arc column heats the near-cathode layer. The reason for this deviation is quite clear: the energy transfer related to electrons cannot be correctly evaluated in the framework of the LTE model.

By integrating the energy flux densities, $q_{\mathrm{hc}}, q_{\mathrm{ec}}$ and $q_{\text {enthalpy }}$, over the cathode surface, we can find powers transported by thermal conduction by heavy particles and by thermal conduction by electrons, net power transported by thermal conduction and power transported by enthalpy transport by the electrons:

$$
\begin{array}{ll}
Q_{\mathrm{hc}}=\int q_{\mathrm{hc}} \mathrm{d} S, & Q_{\mathrm{ec}}=\int q_{\mathrm{ec}} \mathrm{d} S, \\
Q_{\mathrm{c}}=Q_{\mathrm{hc}}+Q_{\mathrm{ec}}, & Q_{\text {enthalpy }}=\int q_{\text {enthalpy }} \mathrm{d} S .
\end{array}
$$

The effect of the arc current on the powers $Q_{\mathrm{hc}}, Q_{\mathrm{ec}}$ and $Q_{\text {enthalpy }}$ is shown in figure 6. One can see that each component of power increases virtually linearly with increasing arc current. The powers transported by thermal 


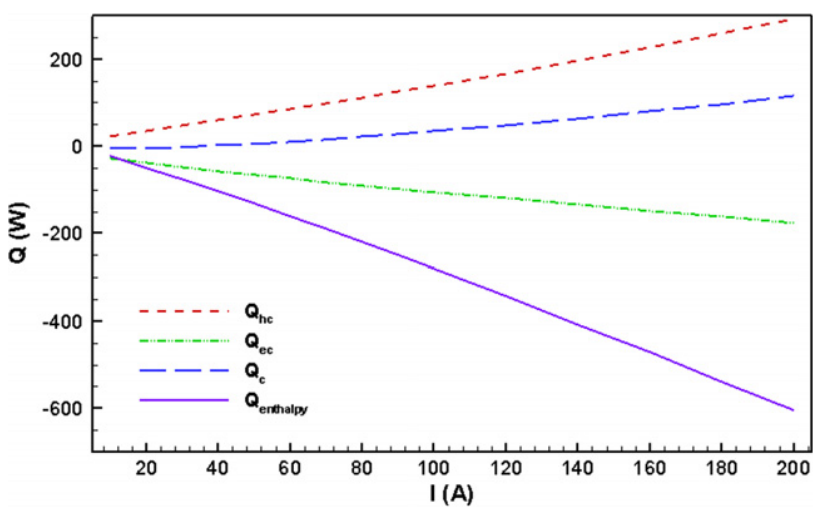

Figure 6. Variations of the total energies delivered to the arc column from the near-cathode layer for different arc currents.

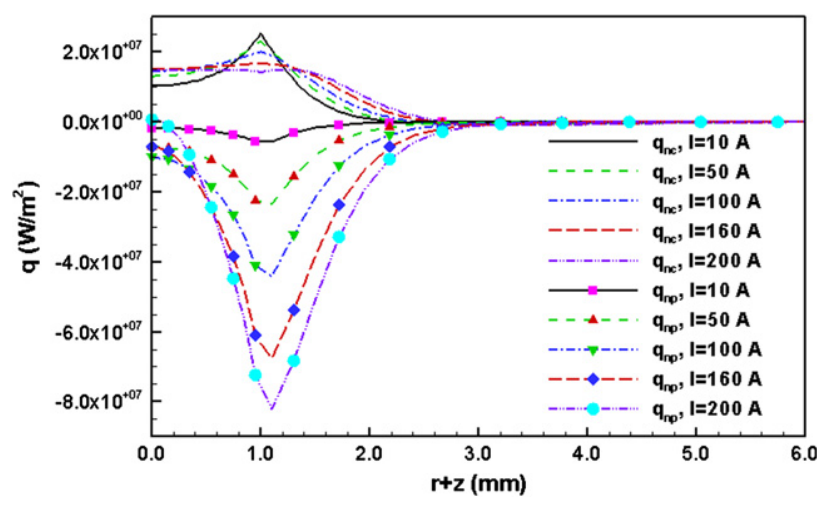

Figure 7. Distributions of the energy flux densities delivered from the near-cathode region to the cathode surface and to the arc column along the cathode surface for different arc currents.

conduction by heavy particles and by thermal conduction by electrons virtually cancel, so the net energy transport due to thermal conduction, $Q_{\mathrm{c}}$, is much smaller than the electron enthalpy transport, $Q_{\text {enthalpy }}$ (also at lower arc current values, where both the absolute values of $Q_{\mathrm{c}}$ and $Q_{\text {enthalpy }}$ are relatively small). In other words, the dominating mechanism of energy transfer from the near-cathode region to the arc column is the electron enthalpy transport. Again, this result supports the corresponding assumption of the model of nonlinear surface heating.

The predicted densities of energy fluxes delivered from the near-cathode layer to the cathode surface and to the arc column for different arc currents (10-200 A) are shown in figure 7. The densities of energy fluxes integrated over the cathode surface (i.e. powers) are shown in figure 8 as functions of the arc current. As the arc current increases, the electric power deposited in the near-cathode layer also increases. The power delivered to the arc column increases with increasing arc current virtually linearly. The power delivered to the cathode surface increases as well, but much more slowly.

\section{Conclusions}

A numerical simulation of a free-burning arc in an atmospheric-pressure argon plasma has been performed in a wide range of arc currents, from 10 to $200 \mathrm{~A}$. The numerical

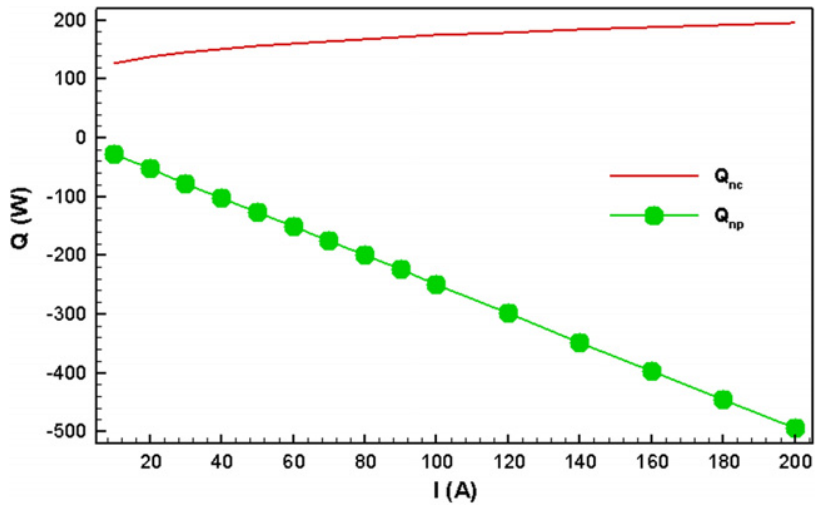

Figure 8. Variations of the total power delivered from the near-cathode layer to the cathode surface and to the arc column with varying arc currents.

model includes modules describing all the components of the system, including the electrodes, except the power supply. In particular, the arc column is modelled by means of a code which is based on a 2-T hydrodynamic model and represents a new version of the FAST-2D code; the cathode body and the near-cathode plasma layer are modelled by means of the free online tool [36], which is based on the model of nonlinear surface heating. Special attention is paid to coupling of these two modules. The main conclusions are as follows.

The electric power deposited into the near-cathode layer is transported not only to the cathode, but also to the arc column. The latter means that the arc column does not heat up the nearcathode plasma region, but rather the other way round: the nearcathode region heats up the arc plasma. This is a very important result confirming the assumption that the cathodic part of the arc discharge is independent of the arc column, which lies at the basis of the model of nonlinear surface heating. The major part of the energy delivered from the near-cathode region to the arc column is due to the electron enthalpy transport. Again, this supports the corresponding assumption of the model of nonlinear surface heating.

Although the LTE model can be employed to predict general features of the heat transfer and flow patterns in the arc column, it does not describe, not even on a qualitative level, the energy exchange between the near-cathode layer and the arc column. The reasons are the following: even in high-current arcs, in which the difference between the electron and heavyparticle temperatures in the bulk of the arc is negligible, there is a part of the arc column adjacent to the near-cathode layer in which the temperatures are essentially different, and a correct description of this part is critical for understanding the heat exchange between the near-cathode layer and the arc column; in previous modelling, due to the neglect of the near-cathode layer, and especially using the LTE model, the predicted gas temperature at the downstream near the cathode tip is always higher than the cathode surface temperature leading to an energy transfer from the arc column to the cathode surface, which is quite different from the results predicted using the non-equilibrium model with the consideration of the nearcathode sheath.

In future work, the influences of the operation conditions, such as different electrode materials and shapes, different plasma-working gases, the contamination of metal vapour 
originating from electrodes, etc, on the arc characteristics need to be studied systematically.

\section{Acknowledgments}

The work was performed within the activities of the project of the National Natural Science Foundation of China (Grant No 10405015) and of the project POCI/FIS/60526/2004 Modes of current transfer to cathodes of high-pressure arc discharges and their stability of FCT, POCI 2010 and FEDER (Portugal). MSB gratefully acknowledges financial support granted by the Tsinghua University towards his research stay in Beijing, China.

\section{References}

[1] Fauchais P and Vardelle A 1997 IEEE Trans. Plasma Sci. 25 1258-80

[2] Gidalevich E, Goldsmith S and Boxman R L 2004 Plasma Sources Sci. Technol. 13 454-60

[3] Keidar M and Schulman M B 2001 IEEE Trans. Plasma Sci. 29 684-9

[4] Keidar M, Fan J, Boyd I G and Beilis I I 2001 J. Appl. Phys. 89 3095-8

[5] Rosén J and Anders A 2005 J. Phys. D: Appl. Phys. 38 4184-90

[6] Jönsson P G, Westhoff R C and Szekely J 1993 J. Appl. Phys. 74 5997-6006

[7] Hsu K C 1982 A self-consistent model for the high intensity free-burning argon arc PhD Thesis Department of Mechanical Engineering, University of Minnesota

[8] Benilov M S 2001 IEEE Trans. Ind. Appl. 37 986-93

[9] Dabringhausen L, Nandelstädt D, Luhmann J and Mentel J 2002 J. Phys. D: Appl. Phys. 35 1621-30

[10] Luhmann J, Lichtenberg S, Langenscheidt O, Benilov M S and Mentel J 2002 J. Phys. D: Appl. Phys. 35 1631-8

[11] Nandelstädt D, Redwitz M, Dabringhausen L, Luhmann J, Lichtenberg S and Mentel J 2002 J. Phys. D: Appl. Phys. 35 1639-47

[12] Lichtenberg S, Nandelstädt D, Dabringhausen L, Redwitz M, Luhmann J and Mentel J 2002 J. Phys. D: Appl. Phys. 35 1648-56

[13] Kettlitz M, Sieg M, Schneidenbach H and Hess H 2005 J. Phys. D: Appl. Phys. 38 3175-81

[14] Dabringhausen L, Langenscheidt O, Lichtenberg S, Redwitz M and Mentel J 2005 J. Phys. D: Appl. Phys. 38 3128-42

[15] Redwitz M, Langenscheidt O and Mentel J 2005 J. Phys. D: Appl. Phys. 38 3143-54

[16] Bötticher R and Kettlitz M 2006 J. Phys. D: Appl. Phys. 39 2715-23

[17] Benilov M S 1998 Phys. Rev. E 58 6480-94

[18] Bötticher R and Bötticher W 2000 J. Phys. D: Appl. Phys. 33 367-74
[19] Coulombe S 2000 Bull. Amer. Phys. Soc. 4518 The 53rd Gaseous Electronics Conf. (Houston, TX) (American Physical Society)

[20] Krücken T 2001 Proc. 9th Int. Symp. on the Science and Technology of Light Sources (Cornell University, Ithaca, August 2001) ed R S Bergman (Ithaca, NY: Cornell University Press) pp 267-8

[21] Bötticher R and Bötticher W 2001 J. Phys. D: Appl. Phys. 34 1110-5

[22] Graser W 2001 Proc. 9th Int. Symp. on the Science and Technology of Light Sources (Cornell University, Ithaca, August 2001) ed R S Bergman (Ithaca, NY: Cornell University Press) pp 211-2

[23] Bötticher R and Bötticher W 2001 Proc. 9th Int. Symp. on the Science and Technology of Light Sources (Cornell University, Ithaca, August 2001) ed R S Bergman (Ithaca, NY: Cornell University Press) pp 207-8

[24] Benilov M S and Cunha M D 2002 J. Phys. D: Appl. Phys. 35 1736-50

[25] Benilov M S and Cunha M D 2003 J. Phys. D: Appl. Phys. 36 603-14

[26] Benilov M S and Cunha M D 2003 Phys. Rev. E 68056407

[27] Bötticher R, Graser W and Kloss A 2004 J. Phys. D: Appl. Phys. 37 55-63

[28] Luijks G M J F, Nijdam S and v Esveld H 2005 J. Phys. D: Appl. Phys. 38 3163-9

[29] Benilov M S, Carpaij M and Cunha M D 2006 J. Phys. D: Appl. Phys. 39 2124-34

[30] Chen X and Li H-P 2001 Int. J. Heat Mass Transfer 44 2541-53

[31] Bott J F 1966 Phys. Fluids 9 1540-7

[32] Mitchner M and Kruger C H Jr 1992 Partially Ionized Gases (New York: Wiley)

[33] Devoto R S 1973 Phys. Fluids 16 616-23

[34] Benilov M S and Marotta A 1995 J. Phys. D: Appl. Phys. 28 1869-82

[35] Benilov M S, Cunha M D and Naidis G V 2005 Plasma Sources Sci. Technol. 14 517-24

[36] http:/www.arc_cathode.uma.pt

[37] Lide D R 2003 The CRC Handbook of Chemistry and Physics 83rd edn (Boca Raton, FL: CRC Press)

[38] Zhu J 1991 Fast-2D: A computer program for numerical simulation of two-dimensional incompressible flows with complex boundaries Report No 690 Institute for Hydromechanics, University of Karlsruhe

[39] Patankar S V 1980 Numerical Heat Transfer and Fluid Flow (New York: McGraw-Hill)

[40] Benilov M S 1999 IEEE Trans. Plasma Sci. 27 1458-63

[41] Maecker H 1960 Z. Phys. 158 392-404

[42] Hsu K C, Etemadi K and Pfender E 1983 J. App. Phys. 54 1293-301

[43] Hsu K C and Pfender E 1983 J. Appl. Phys. 54 4359-66

[44] Charrada K and Zissis G and Aubes M 1996 J. Phys. D: Appl. Phys. 29 2432-8

[45] Haidar J 1999 J. Phys. D: Appl. Phys. 32 263-72 